\title{
Bed occupancy measurements using under mattress pressure sensors for long term monitoring of community-dwelling older adults
}

\author{
Matthew Taylor ${ }^{1}$, Member, IEEE, Theresa Grant ${ }^{2,3}$, Frank Knoefel ${ }^{1,2}$, Rafik Goubran ${ }^{1,2}$, Fellow, IEEE \\ 1. Department of Systems and Computer Engineering. Carleton University. Ottawa, Canada \\ 2. Bruyère Research Institute. Bruyère Continuing Care. Ottawa, Canada \\ 3. School of Rehabilitation Sciences. University of Ottawa. Ottawa, Canada
}

\begin{abstract}
There is a growing demand for systems that support independent living into advanced age. Technologies that monitor changes in the amount of time older adults spend in bed have the potential to detect critical changes in mobility and support earlier health intervention. Although under mattress sensors have been used previously, processing algorithms were designed for short term monitoring. The objective of this paper was to develop an algorithm and determine optimal sampling rate to obtain bed occupancy characteristics over the longer term. Under mattress sensors were installed in the home of an older adult and data collected over a 3 month period. A processing algorithm was developed to extract bed occupancy information including time in bed, number of bed exits and time of first morning exit. Data were compared using various sampling rates and processing times. Findings indicate that the ideal down sample time for the application was 5 seconds $(0.2 \mathrm{~Hz})$ and that computational time requirements could be reduced significantly without sacrificing the ability to accurately measure the total time spent in bed and number of bed exits. Features of bed occupancy were plotted and patterns discovered which may be of interest to health clinicians and sleep researchers.
\end{abstract}

Keywords - pressure sensors; patient monitoring; bed occupancy; data processing; down sampling

\section{INTRODUCTION}

The population of Canadians over the age of 65 years will rise from $13.9 \%$ in 2011 to $22.8 \%$ in 2036 [1]. There is evidence that ageing at home rather than in an institution is desirable from both an individual perspective [2] as well as a cost-saving system perspective [3]. Given this preference along with observed trends [4], there is a need to develop systems and technologies to support the ability of older adults to live independently in their homes for as long as safely possible. Remote monitoring technologies have the potential to do this by detecting changes related to health or mobility patterns and serving as early warning systems. Caregivers or health providers could therefore be alerted to intervene in order to prevent a critical health event.

The time that individuals spend in bed and the difficulty

This work was funded by the Natural Sciences and Engineering Research Council (NSERC), the Canadian Institutes of Health Research (CIHR) and Carleton University they have rising from bed have been linked to various aspects of health status and independence [5-7]. Thus, being able to monitor bed occupancy with smart sensors has relevance for developing technologies capable of detecting critical changes in health status in an unobtrusive way.

This paper describes the use and development of a type of smart sensor technology with the aim of measuring bed occupancy characteristics in a community-dwelling older adult over a 3 month period.

\section{BACKGROUND}

Patient monitoring is a particularly active field of research with many new technologies appearing regularly, from wearable ECG monitoring devices that can monitor cardiac information while the patient performs their daily activities [8], [9] to motion sensors that can monitor activity in one area of the home. Pressure sensors installed under the mattress can monitor bed occupancy. The amount of time the person spends in bed, the number of times they get up in the night, and the time of day they sleep are all features of bed occupancy that can be extracted from pressure sensors [10].

Past research on pressure sensors has shown that this type of technology can be used to determine bed occupancy, calculate sit-to-stand times and breathing rates [11-12] using short term recordings. However, algorithms used in this previous work would not be feasible to use for long term recordings without segmentation to isolate useable sections of data for the algorithms.

To monitor bed occupancy over the long term, methods are required that allow accurate but time-efficient processing of a large amount of data. Although bed occupancy information has been collected and interfaces designed to display the information in a meaningful way to health care providers [10], the problem of feasible data processing currently exists as a potential barrier to the adoption of this technology. This paper aims to address this problem by examining the minimum data sampling rate required to calculate accurate and time-efficient measures of bed occupancy. 


\section{TECHNOLOGY}

Bed occupancy can be measured using various technologies, IR motion detectors or video cameras could be placed above the bed, detecting bed occupancy [13]. Strain gauges could measure the flex of the mattress as the person enters it. Force plates could be placed under the bed legs measuring the applied weight. We proposed the use of fiber optic pressure sensors embedded in a mat placed under the mattress. The pressure sensors will detect the change in pressure as the person enters or exits the bed. This type of technology is far less obtrusive to the habitant compared to video cameras and captures the pressure signal in more detail compared to strain gauges or force plates through its many sensors.

The bed mat shown in Figure 1 is placed under the mattress in the person's home and connects to a Bluetooth transmitter.

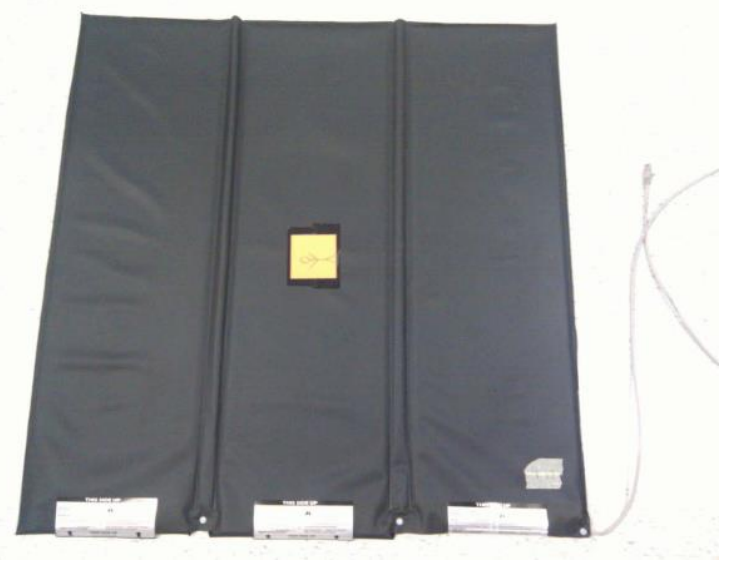

Figure 1. S4 Sensors Inc. Under-Mattress Pressure Sensor.

Data from the bed mat is captured by a low-end Dell OptiPlex PC. Novel signal processing algorithms were developed in MATLAB (MathWorks, Inc. Mass. USA) to process the long term bed mat recordings.

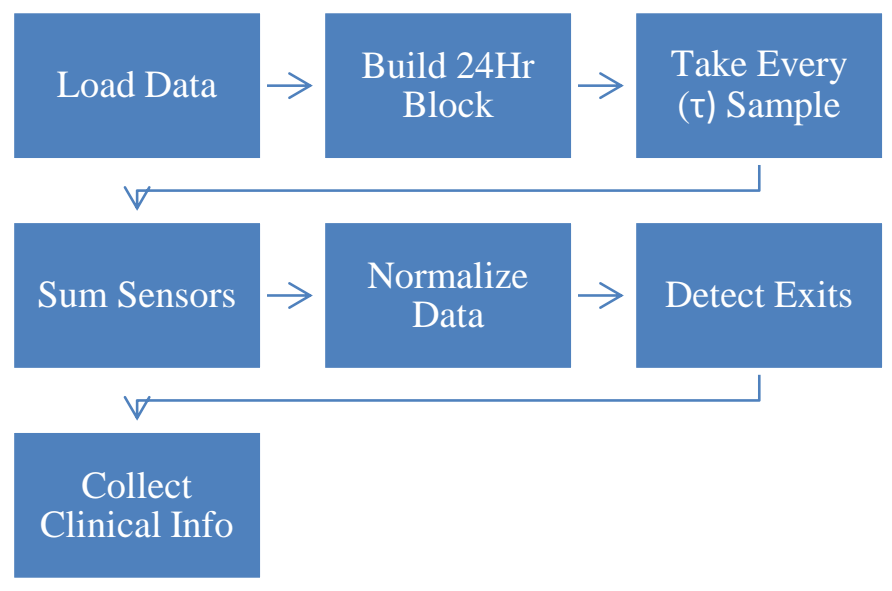

Figure 2. Flowchart of processing required for bed occupancy measurement system.

\section{MEASUREMENTS}

The bed mat records pressure measurements using 72 equally spaced sensors in an $8 \times 9$ grid array. The sensors are sampled at a rate of $20 \mathrm{~Hz}$. The raw data is saved into CSV files on the PC in one hour blocks. Using the custom processing algorithm, bed occupancy information is extracted from the data.

Occupancy is determined when the observed pressure reaches a minimum threshold. The threshold is configured based on the size of the person. The individual sensor values are summed to give a total measurement of pressure in the bed. This allows occupancy detection regardless of where the patient enters or exits the bed. We are able to extract several clinically relevant characteristics of bed occupancy; the number of hours spent in bed, the number of exits in the night, and what time the person got up in the morning.

The high sampling rate and large number of sensors creates a very large data set requiring vast computational resources. To reduce the processing time the sampling rate was varied.

\section{EXPERIMENT}

The under mattress pressure sensor was installed in the home of an older adult who consented to participate in the study. Data was collected continuously for 3 months and will continue for up to one year.

Using the algorithms developed in MATLAB the data was segmented into 24 hour blocks from 12PM - 12PM, capturing the full night activity. The 24 hour blocks were processed and the following bed occupancy information was extracted:

- Total time in bed (Hours)

- Total time out of bed (Hours)

- Time of day when exiting bed

- Number of bed exits

The information can be observed night by night or can be graphed to display long term trends in bed occupancy. We use examples of both types of displays in this paper. Further research is being conducted to determine optimal display of bed occupancy information for clinicians.

The dataset was processed using the 'full power' algorithm. This used the full sampling rate and considered all 72 sensors in measuring bed occupancy. These measures will be used as a comparison to the measures using the 'low power' algorithms.

Since the bed mat itself could not be modified, the effects of a reduced sampling rate were measured by considering a pressure measurement every $\tau$ seconds from the recorded data. The value of $\tau$ was varied from 0.05 to 600 seconds with several steps in between; effectively a sampling rate from 20 to $0.0016 \mathrm{~Hz}$. 
With a fixed sampling rate the processing time can vary based on the nature of the data set. If there is very little bed activity processing is much quicker than data showing several bed exits. To gain the most practical results from the experiment the sampling rate was varied in-line with the processing algorithm. A timestamp was placed at the beginning of the algorithm and one at the end of processing to allow us to measure the processing duration.

\section{RESULTS}

The sampling rate was varied in-line with the processing algorithm used for extracting bed occupancy measurements. A small section of data consisting of one week is presented in the results. The observed processing times for each sampling rate are presented in Table 1. Since we conducted the experiment in-line with the rest of the algorithm we found that there was a limit to the payoff of reducing the sampling rate. When taking a sample from every 5 seconds to every 600 seconds the processing time was roughly the same. Figure 3 is a visual representation of the processing time per sampling rate. The processing time grew exponentially as it approached 'full power' therefore logarithmic representation was used.

Table 1. Effects of reduced sampling rate.

\begin{tabular}{|r|r|r|}
\hline $\begin{array}{c}\text { Sample Time } \\
\tau(\mathrm{sec})\end{array}$ & $\begin{array}{c}\text { Sampling Rate } \\
\mathrm{f}(\mathrm{Hz})\end{array}$ & \multicolumn{1}{l|}{ Duration } \\
\hline $5-600$ & $0.2-0.002$ & $0: 04: 49-0: 05: 23$ \\
\hline 1 & 1 & $0: 08: 20$ \\
\hline 0.5 & 2 & $0: 11: 57$ \\
\hline 0.4 & 2.5 & $0: 14: 00$ \\
\hline 0.3 & 3.3 & $0: 19: 31$ \\
\hline 0.2 & 5 & $0: 35: 14$ \\
\hline 0.1 & 10 & $1: 59: 27$ \\
\hline 0.05 & 20 & $8: 08: 19$ \\
\hline
\end{tabular}

Processing Time Vs. Sample Time

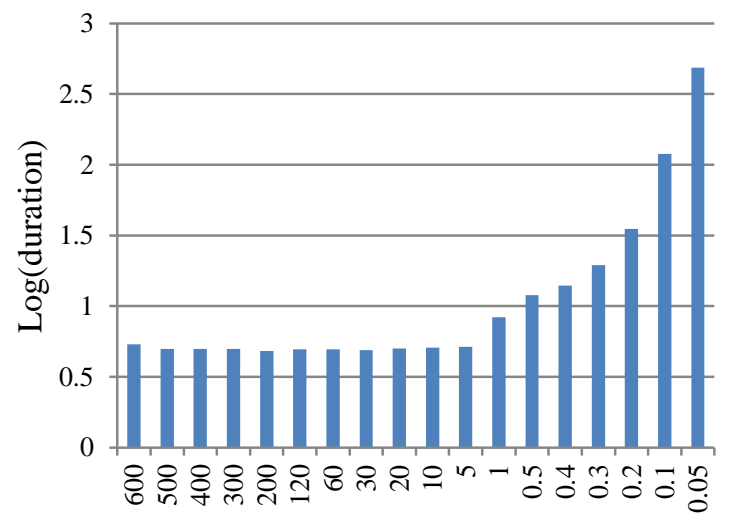

Sample Time $\tau$ (sec)

Figure 3. Processing time of algorithm for down-sampled time $(\tau)$.
The bed occupancy features for one week were compared to determine whether any errors occurred due to down sampling. Figure 4 shows the total hours spent in bed per day for each of the sample rates. Each plot follows the 'full power' one; however there is some loss in accuracy. At the highest down sample rate, 600 seconds, we found the error in bed occupancy time was between $45-90$ minutes per day. The error quickly decreased as we increased the sampling rate. The error for a sample time of 5 seconds $(0.2 \mathrm{~Hz})$ was 0 - 30 seconds per day which is reasonably good accuracy and required roughly the same processing time as the highest down sample rate tested.

Another aspect of bed occupancy - number of bed exits - is shown in Figure 5. The number of exits detected for each day remained true for almost all of the sampling rates tested. The only exception was the highest down sample rate, 600 seconds, when it incorrectly detected exits 4 out of the 7 days observed.

Based on the observed processing times and considering the errors that could exist at the limit of low sampling we determined the ideal down sample time for our application was 5 seconds $(0.2 \mathrm{~Hz})$. The processing time required was very low, the correct number of exits was determined, and there was less than 30 seconds of error in the bed occupancy measure per day.

The full 3 month dataset, was subsequently processed using the algorithm, set at $0.2 \mathrm{~Hz}$ sampling. Bed occupancy measurements were taken over the 3 month recording period and the total time in bed per day was measured. Figure 6 illustrates the distribution of bed occupancy times. By observing this plot we can see there are a few outliers which may represent either under sleeping (low bed occupancy) or over sleeping (high bed occupancy). Table 2 summarizes the outliers: the number of nights with more than 12 hours occupancy, less than 6 hours occupancy, and the combined nights. This allows researchers to compare these problem nights to the number of normal occupancy nights.

\section{Bed Occupancy Per Day}

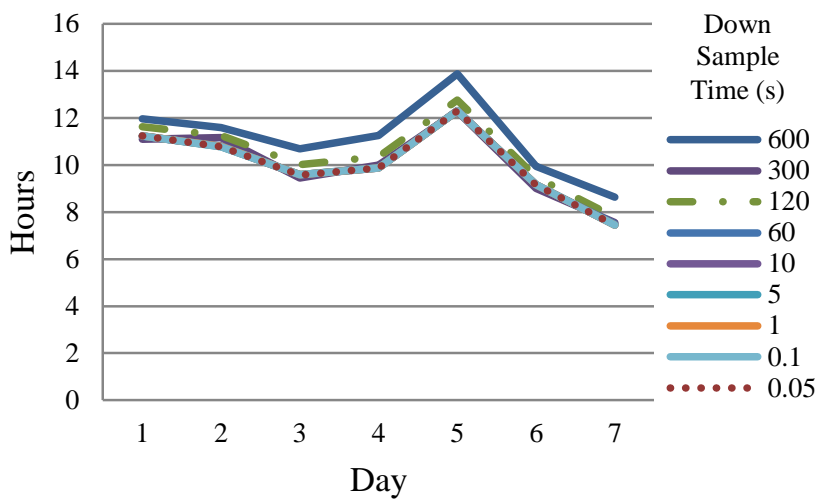

Figure 4. Measured bed occupancy time per sampling rate. 


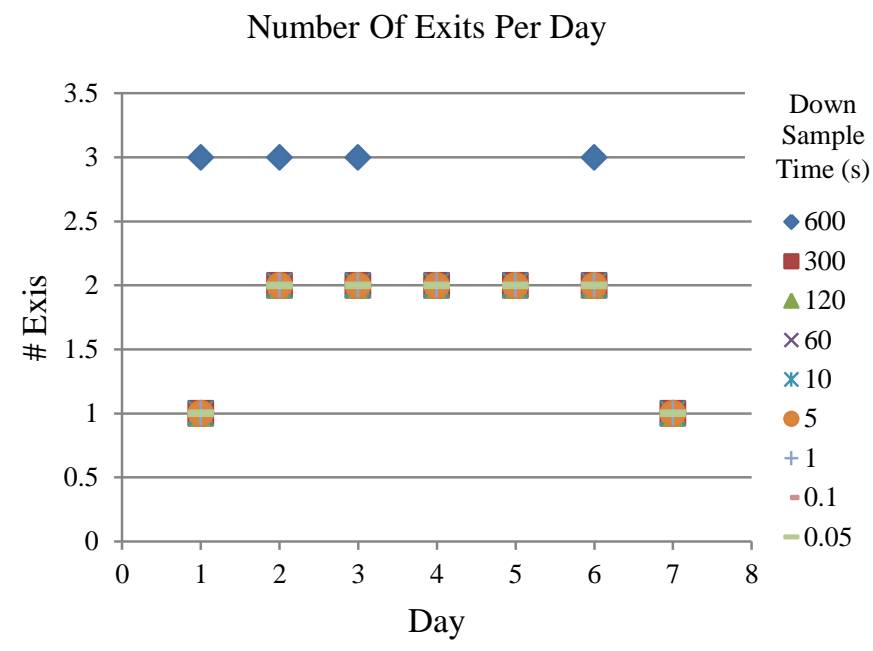

Figure 5. Number of exits detected per sampling rate.

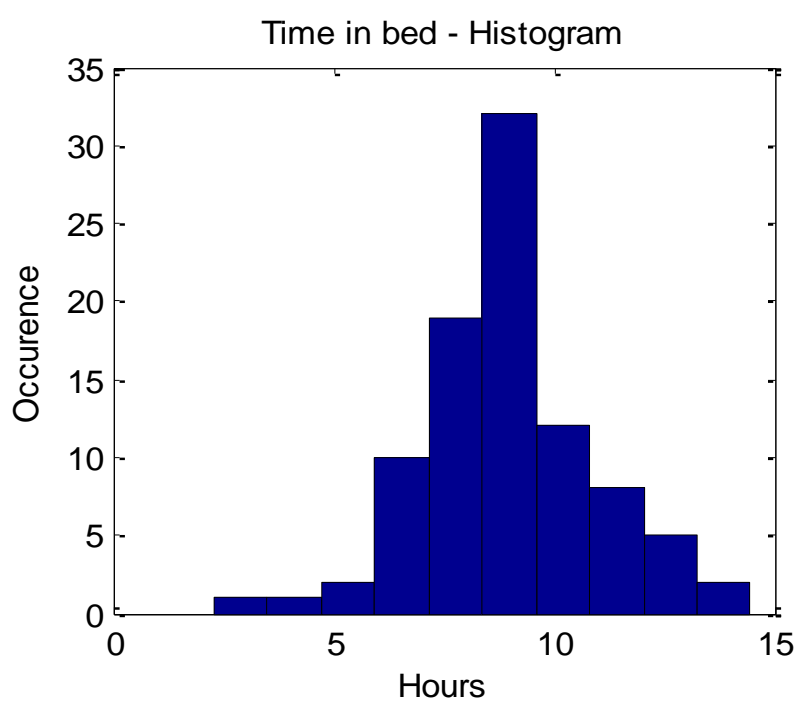

Figure 6. Distribution of bed occupancy time over 3 months.

Table 2. Bed occupancy patterns

\begin{tabular}{|l|l|l|}
\hline & Nights & \% over all \\
\hline High occupancy $(>12 \mathrm{Hrs})$ & 7 & 7.6 \\
\hline Low occupancy $(<6 \mathrm{Hrs})$ & 4 & 4.3 \\
\hline Combined $(<6 \&>12)$ & 11 & 12.0 \\
\hline Normal $(6-12 \mathrm{Hrs})$ & 81 & 88.0 \\
\hline Total nights captures & 92 & 100 \\
\hline
\end{tabular}

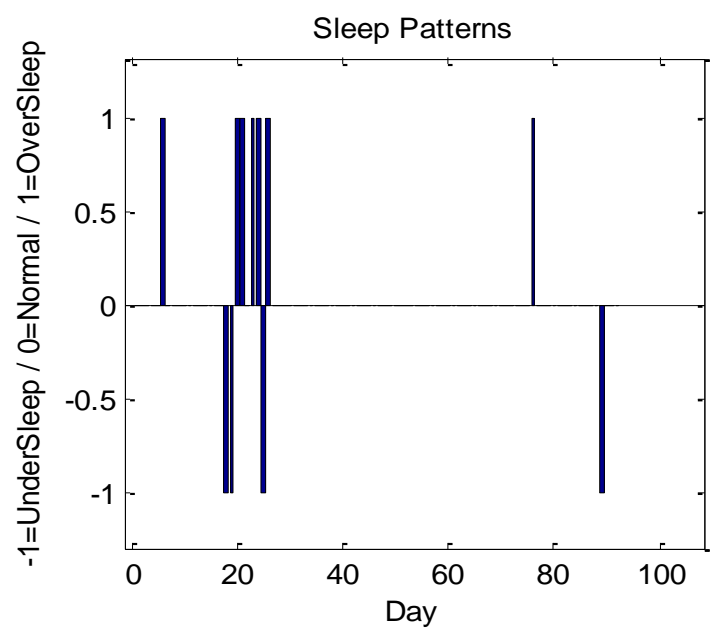

Figure 7. Abnormal bed occupancy observed.

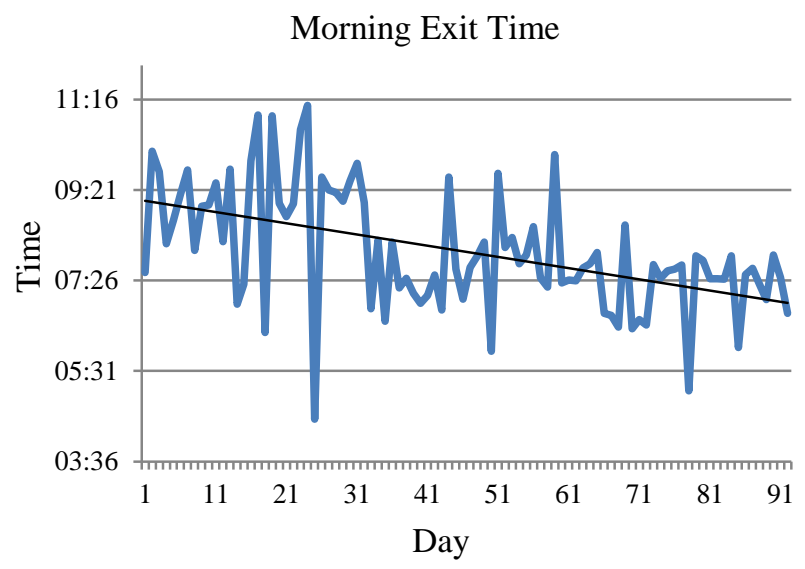

Figure 8. Morning bed exit time over 3 months.

\section{DISCUSSION}

A key finding of this study was that using a sample every 5 seconds instead of every 0.05 seconds reduced the number of calculations by 100 times. This drastically reduced the computational time required by the algorithm, from 8 hours to 5 minutes for one week of data. This significant difference in processing time, without sacrificing accuracy, is important since it indicates that the collection of bed occupancy data is feasible for the purpose of long-term bed occupancy monitoring.

The measurement error we calculated of up to 30 seconds per day is small in comparison to the range of morning exit times and time spent in bed. Thus the sampling rate identified is associated with an acceptable level of accuracy with respect to the parameters measured. When performing bed occupancy measures for long term patient monitoring fine details like the exact time of bed exit within less than a 30 second margin error may not be necessary. This would be particularly true if the purpose of monitoring is to observe changes in a pattern. 
It is important to note, however, that if the timing of the bed transfer is of interest, then another algorithm would need to be used. The time it takes to rise from the sitting position is approximately 5 seconds or less [11]. This period of time is much shorter that the margin of error calculated for the algorithm used in this study. In this case the 'low power' algorithm for detecting bed occupancy can be used to roughly determine when a sit-to-stand transfer occurs. The algorithm could then switch to 'full power' mode to accurately determine the sit-to-stand time and then switch back to 'low power' to continue conserving resources. This hybrid algorithm is part of our current research efforts in long term health monitoring.

With respect to the occupancy patterns observed, an interesting discovery was made when plotting the data longitudinally; this is shown in Figure 7. Most of the problem nights occurred within the first month of data collection. There was a concentration of low bed occupancy nights followed by several high occupancy nights. These periods of high and low occupancy may have been associated with over and under sleeping or a number of potential health conditions such as depression or painful syndromes. The pattern observed in this study could be of interested to a clinician who is monitoring the patient. For example, if a physician prescribes a change in medication, the effects on sleep patterns could be monitored more accurately.

An individual's morning exit time can vary significantly [14]. However when plotting the morning exit time for this participant over the 3 month recording period, as shown in Figure 8, another interesting trend was discovered. There was much greater variability in the first month, concentrated around the time of the high and low bed occupancy. The variability slowly decreased and the time of morning exits became earlier over time. Future research needs to be done on establishing ranges of normal rising time and effect on older adult health. The current study provides more feasible and accurate means of doing so.

\section{CONCLUSION}

This study demonstrates that long term monitoring of bed occupancy using under mattress bed sensors is possible using a low sampling rate. The ideal sampling rate in terms of time efficient and accurate processing was $0.2 \mathrm{~Hz}$. The error associated with this sampling rate did not compromise the collection of accurate bed occupancy data.

Data patterns observed in this 3 month study revealed a period of high variability in bed occupancy followed by stabilization. The ability to make these types of observations gives researchers new avenues to monitor critical changes in sleep patterns and examine how they may be associated with health status.

Future algorithm development for the purpose of monitoring aspects of bed mobility among older adults will need to integrate low sampling rates to process large amounts of data with higher sampling rates that can accurately measure high precision events such as sit-to-stand. This integration will make it feasible to accurately process large amounts of data over the long term without losing the ability to process the fine details when required.

\section{REFERENCES}

[1] Statistics Canada, "Population projections for Canada, Provinces and Territories, 2009-2036," Statistics Canada, Ottawa, ON, 91-520-X, Jun. 2010.

[2] J. Wiles, A. Leibing, N. Guberman, J. Reeve, R. Allen, "The Meaning of "Ageing in Place" to Older People," Gerontologist, vol. 52, pp. 357-366, 2012.

[3] N. Chappell, B. Dlitt, M. Hollander, J. Miller, C. McWilliam, "Comparative Costs of Home Care and Residential Care," Gerontologist, vol. 44, pp. 389-400, Jun. 2004.

[4] Ontario's Local Health Integration Networks, "Aging at Home Strategy" [Online], Dec. 20, 2012. http://www.lhins.on.ca/page.aspx $\mathrm{id}=880$

[5] N. Alexander, J. Grunawalt, S. Carlos, J. Augustine, "Bed mobility task performance in older adults," J. Rehabil Res Dev, vol. 37, pp. 633-638, Sept. 2000.

[6] A. Cieza, T. Brockow, T. Ewert, E. Amman, B. Kollerits, "Linking health-status measurements to the international classification of functioning, disability and health," $J$ Rehabil Med, vol. 34, pp. 205-210, 2002.

[7] K. Wells, A. Stewart, R. Hays, M. Burnam, W. Rogers, M. Daniels, S. Berry, S. Greenfield, J. Ware, "The functioning and well-being of depressed patients. Results from the Medical Outcomes Study," JAMA, vol. 262, pp. 914-919, Aug. 1989.

[8] P. Bifulco, M. Cesarelli, A. Fratini, M. Ruffo, G. Pasquariello, G. Gargiulo, "A wearable device for recording of biopotentials and body movements," in Proc. of IEEE International Symposium on Medical Measurements and Applications, Bari, Italy, 2011, pp. 469-472.

[9] E. Sardini, M. Serpelloni, M. Ometto, "Multi-parameters wireless shirt for physiological monitoring," in Proc. of IEEE International Symposium on Medical Measurements and Applications, Bari, Italy, 2011, pp. 316-321.

[10] M. Pouliot, V. Joshi, R. Goubran, F. Knoefel, "Bed occupancy monitoring: Data processing and clinician user interface design," in Proc. of IEEE International Conference of the Engineering in Medicine and Biology Society, San Diego, USA, 2012, pp. 5810-5814.

[11] A. Arcelus, I. Veledar, R. Goubran, F. Knoefel, H. Sveistrup, M. Bilodeau, "Measurements of Sit-to-Stand Timing and Symmetry From Bed Pressure Sensors," IEEE Trans. Instrumentation and Measurement, vol. 60, pp. 1732-1740, May 2011.

[12] M. Holtzman, A. Arcelus, R. Goubran, F. Knoefel, "Breathing Signal Fusion in Pressure Sensor Arrays," in Proc. of IEEE International Workshop on Medical Measurements and Applications, Ottawa, Canada, 2008, pp. 71-76.

[13] M. Berger, A. Armitage, "Room occupancy measurement using low-resolution infrared cameras," in Proc. of IET Irish Signals and Systems Conference, Cork, Ireland, 2010, pp. 249-254.

[14] E. Klerman, D. Dijk, "Age-related reduction in the maximal capacity for sleep - implications for insomnia," Current Biology, vol. 18, pp. 1118-1123, Aug. 2008. 